\title{
Feedback Linearization and Control Design for Nonlinear Descriptor Systems
}

\author{
Elvis Mayk Chaves Barbosa* Fernando de Oliveira Souza ** \\ Reinaldo Martinez Palhares** \\ * Graduate Program in Electrical Engineering - Universidade Federal \\ de Minas Gerais - Av. Antônio Carlos 6627, 31270-901, Belo \\ Horizonte, $M G$, Brazil, \\ (e-mail: elvismayk@ufmg.br). \\ ** Department of Electronics Engineering - Universidade Federal de \\ Minas Gerais - Av. Antônio Carlos 6627, 31270-901, Belo Horizonte, \\ MG,Brazil (e-mails: fosouza@cpdee.ufmg.br; rpalhares@ufmg.br)
}

\begin{abstract}
This paper deals with the stabilization problem of continuous-time nonlinear descriptor systems. The methodological contribution is to propose a state transformation based on a canonical controllable form, originally proposed for linear descriptor systems, such that a feedback linearizable nonlinear descriptor model can be achieved and, consequently, the control law design designed. The closed-loop stability is checked in the sense of the standard Lyapunov theory. Two examples are presented to illustrate details of implementation. The concluding remarks discuss about the effectiveness and drawbacks of the proposed strategy.
\end{abstract}

Keywords: Nonlinear Descriptor Systems, Feedback Linearization, Canonical Controllable Form.

\section{INTRODUCTION}

Descriptor systems, also know as singular systems or differential/algebraic systems (Luenberger, 1978) are a class of systems for which its dynamics can be modeled by the descriptor state-space representation. The descriptor system representation is able to characterize differential algebraic equations, therefore it is able to model further structure properties of physical phenomenons and more general systems than the standard state-space representation (Yang et al., 2013b). As a consequence, the descriptor representation is frequently used to model mechanical systems (Blandeau et al., 2018), (Guelton et al., 2008), robots (Schulte and Guelton, 2009) and electrical circuits (Newcomb and Dziurla, 1989). Descriptor systems have been investigated extensively since Luenberger (1978) introduced its time-invariant case.

The feedback linearization is a technique used in control of nonlinear systems which consists in transforming an original system model into an equivalent one of a simpler form (Slotine and $\mathrm{Li}, 1991$ ). However, few works investigated the application of feedback linearization in nonlinear descriptor systems. Kawaji and Taha (1994) deals with this problem in a specific class of descriptor systems, without using a state realization method. Boukas and Habetler (2003) presents exact feedback linearization with state derivative feedback applied to control of a fieldoriented induction motor. In Spong (1994) is presented a partial feedback linearization for underactuated mechanical system which can be written as descriptor systems. In Xiaoping (1993) is presented how the exact linearization of nonlinear descriptor systems can be performed by using a change of state coordinates. Although these works have investigated transformations in the context of descriptor systems, there is not still a count part for presenting conditions for designing nonlinear controllers per si.

Motivated by the lack of investigation about the feedback linearization of descriptor systems and its control design, this work proposes to use a change of state coordinates for get a feedback linearizable nonlinear descriptor model. The obtained model has a specified controllable form with certain properties and the proposed control law is obtained in a follow up way along the process of transformation. Also, the conditions to use the transformations are presented and the stability of the closed-loop system is validated after the controller design. Examples of application illustrate the process how the control law is obtained and numerical simulations show its effectiveness.

The paper is organized as follows: in Section 2 is presented the notation and the theoretical foundation around the technique of feedback linearization presented in Section 3. Numerical application examples of the proposed method are presented in Section 4 with discussions of the results. Discussions and relevant questions are stated in 5. Finally, Section 6 concludes this work.

\section{PRELIMINARIES AND NOTATION}

This work deals with continuous-time nonlinear descriptor models of the form:

$$
E(x(t)) \dot{x}(t)=A(x(t)) x(t)+B(x(t)) u(t)
$$

where $E(x(t)) \in \mathbb{R}^{n \times n}, A(x(t)) \in \mathbb{R}^{n \times n}, B(x(t)) \in$ $\mathbb{R}^{n \times m}, x(t)=\left[x_{1}, \ldots, x_{n}\right]^{T} \in \mathbb{R}^{n}$ is the descriptor vector, $u(t)=\left[u_{1}, \ldots, u_{m}\right]^{T} \in \mathbb{R}^{m}$ is the input vector. The 
matrix $E(x(t))$ can be singular (Luenberger, 1978), it is smooth with respect to $x$ and it has constant rank. The uniqueness and existence of solutions for system $(E(x(t)), A(x(t)), B(x(t)))$ is guaranteed if $\operatorname{det}(\lambda E-A) \neq$ 0 for some $\lambda \in \mathbb{C}$ and, then the pair $(E, A)$ is regular (Dai, 1989). According Yang et al. (2013a), a regular descriptor system can present impulsive jumps in its response which are undesirable and could completely destroy the system. The existing solution is impulsive-free if $\operatorname{deg}(\operatorname{det}(\lambda E-$ $A))=\operatorname{rank}(E)$, for any initial condition. From this point, the time $t$ argument of the signals is omitted.

A nonlinear descriptor system of the form in (1) can be written as

$$
E(x) \dot{x}=w(x, u)=f(x)+g(x) u
$$

where $f(x)$ and $g(x)$ are smooth with respect to $x$ and $u$, respectively. In order to have a set up for transform the form of this system, we introduce the following definition: Definition 1. (Xiaoping, 1993). A system as in (2) is said to be state-equivalent at $x_{0}$ to the system of the form

$$
E^{\prime}(z) \dot{z}=w^{\prime}(z, u)
$$

if there exist an open neighborhood $D$ of $x_{0}$, a nonsingular matrix function $P(x)$ and a diffeomorphism $z=T(x)$, $T: D \rightarrow T(D)$, such that

$$
\begin{gathered}
P(x) E^{\prime}(T(x)) \frac{\partial T(x)}{\partial x}=E(x) \\
P(x) w^{\prime}(T(x), u)=w(x, u)
\end{gathered}
$$

\subsection{Useful Controllability results}

Consider a linear descriptor system of the form

$$
E \dot{z}=A z+B v
$$

with $z \in \mathbb{R}^{n}$ and $v(t) \in \mathbb{R}^{m}$. Thus the following lemmas are useful.

Lemma 2. (Helmke and Shayman (1989)). A system $(E, A, B)$ in (5) given as the standard form in (6) is controllable if and only if the subsystems $\left(A_{1}, B_{1}\right)$ and $\left(A_{2}, B_{2}\right)$ are controllable.

$$
\begin{aligned}
& \dot{z}_{1}=A_{1} z_{1}+B_{1} v \\
& A_{2} \dot{z}_{2}=z_{2}+B_{2} v
\end{aligned}
$$

where $A_{2}$ is nilpotent, $B_{1} \in \mathbb{R}^{r \times m}$ and $B_{2} \in \mathbb{R}^{(n-r) \times m}$. $z_{1} \in \mathbb{R}^{r}$ and $z_{2} \in \mathbb{R}^{(n-r)}$ are divisions of state vector $z$ and $r \triangleq \operatorname{deg} \operatorname{det}(\lambda E-A)$.

The form in (6) is called standard decomposition and the subsystems (6a) and (6b) are called slow and fast subsystems, respectively.

Lemma 3. (Xiaoping (1993)). Any controllable linear descriptor system of the form in (5) with $\operatorname{rank}(B)=m$ is state-equivalent to the system (6) in the canonical form:

$$
\begin{aligned}
A_{1} & =\left[\begin{array}{ccc}
A_{11} & \ldots & A_{1 m} \\
\vdots & \ddots & \vdots \\
0 & \ldots & A_{m m}
\end{array}\right] \quad B_{1}=\operatorname{diag}\left(b_{1}, \ldots, b_{m}\right) \\
A_{2} & =\left[\begin{array}{ccc}
\hat{A}_{11} & \ldots & \hat{A}_{1 m} \\
\vdots & \ddots & \vdots \\
0 & \ldots & \hat{A}_{m m}
\end{array}\right] \quad B_{2}=\operatorname{diag}\left(\hat{b}_{1}, \ldots, \hat{b}_{m}\right)
\end{aligned}
$$

with the following elements,

$$
\begin{gathered}
A_{i i}=\left[\begin{array}{cccc}
0 & \ldots & 0 & c_{i i}^{1} \\
1 & \ldots & 0 & c_{i i}^{2} \\
\vdots & \ddots & \vdots & \vdots \\
0 & \ldots & 1 & c_{i i}^{K_{i}}
\end{array}\right]_{K_{i} \times K_{i}} \quad i \in\{1, \ldots, m\} \\
A_{i j}=\left[\begin{array}{cccc}
0 & \ldots & 0 & c_{i j}^{1} \\
0 & \ldots & 0 & c_{i j}^{2} \\
\vdots & \ddots & \vdots & \vdots \\
0 & \ldots & 0 & c_{i j}^{K_{i}}
\end{array}\right]_{K_{i} \times K_{j}}^{2} \quad i, j \in\{1, \ldots, m\} \text { e } i<j \\
\hat{A}_{i i}=\left[\begin{array}{cccc}
0 & \ldots & 0 & 0 \\
1 & \ldots & 0 & 0 \\
\vdots & \ddots & \vdots & \vdots \\
0 & \ldots & 1 & 0
\end{array}\right]_{L_{i} \times L_{i}} \quad i \in\{1, \ldots, m\} \\
\hat{A}_{i j}=\left[\begin{array}{llll}
0 & \ldots & 0 & d_{i j}^{1} \\
0 & \ldots & 0 & d_{i j}^{2} \\
\vdots & \ddots & \vdots & \vdots \\
0 & \ldots & 0 & d_{i j}^{L_{i}}
\end{array}\right]_{L_{i} \times L_{j}}^{2} \quad i, j \in\{1, \ldots, m\} \text { e } i<j \\
b_{i}=\left[\begin{array}{l}
1 \\
\vdots \\
0
\end{array}\right] \hat{b}_{i}=\left[\begin{array}{l}
K_{i} \times 1 \\
\vdots \\
0
\end{array}\right] L_{i} \times 1
\end{gathered}
$$

where $c_{i j}^{k}$ and $d_{i j}^{k}$ are real numbers. $K_{i} \in \mathbb{N}$ and $L_{i} \in \mathbb{N}$ are controllability indices ${ }^{1}$ of the slow and fast subsystems, respectively, with $K_{1}+\cdots+K_{m}=r$ e $L_{1}+\cdots+L_{m}=n-r$.

\section{METHOD}

Based on the definitions of canonical linear descriptor systems described in Lemmas 2 and 3, we can state that a controllable linear descriptor system could have the form:

$$
E_{c} \dot{z}=A_{c} z+B_{c} v
$$

with the following structures for the matrices:

$$
\begin{array}{rlr}
E_{c} & =\left[\begin{array}{cc}
I_{r} & 0_{r,(n-r)} \\
0_{(n-r), r} & A_{2}
\end{array}\right] & B_{c}=\left[\begin{array}{l}
B_{1} \\
B_{2}
\end{array}\right] \\
A_{c}=\left[\begin{array}{cc}
A_{1} & 0_{r,(n-r)} \\
0_{(n-r), r} & I_{(n-r)}
\end{array}\right] &
\end{array}
$$

We propose a simpler form for nonlinear descriptor systems as in (1) in the following definition:

Definition 4. (Normally feedback linearization). Consider a system in the form (2), where $E(x), f(x)$ and $g(x)$ are continuously differentiable in domain $D$. (2) is said to be normally feedback linearizable if it is state-equivalent to a system in the form:

$$
E_{c} \dot{z}=A_{c} z+B_{c} \Gamma(x)[u-\alpha(x)]
$$

with $E_{c}, A_{c}$ and $B_{c}$ defined in (10) and $\Gamma(x) \in \mathbb{R}^{m \times m}$ is nonsingular for all $x \in D$ and $\alpha(x) \in \mathbb{R}^{m \times 1}$.

\footnotetext{
1 Also called Hermite indices. Let $(A, B)$ be a controllable system of order $n$ and let $b_{1}, \ldots, b_{m}$ denote colums of $B$. Delete in the controllability matrix of $(A, B)$, while going from left to right, all vectors $A^{k} b_{l}$ which are linearly dependent on the set of all previous vectors. The remaining vectors

$$
T_{A B} \triangleq\left(b_{1}, \ldots, A^{\left(K_{1}-1\right)} b_{1}, \ldots, b_{m}, \ldots, A^{\left(K_{m}-1\right)} b_{m}\right)
$$

form a basis of the space $\mathbb{C}^{n}$ with nonnegative integers $K_{1}, \ldots, K_{m}$ satisfying $K_{1}+\cdots+K_{m}=n$. This set of numbers are called controllability indices of $(A, B)$ (Helmke and Shayman, 1989).
} 
Thus, considering the normally feedback linearizable system as in (11), the following control law reduces the original system to a linear descriptor system as in (9):

$$
u(t)=\Gamma^{-1}(x) v(z)+\alpha(x)
$$

where $v(z)$ is the control law for the linear system in the new state coordinate $z$. The closed-loop of system (1) with control law as in (12) can be written as

$$
E(x) \dot{x}=A(x) x+B(x) \Gamma^{-1}(x) v(z)+B(x) \alpha(x)
$$

Now, the main proposition can be presented.

Proposition 5. A nonlinear descriptor system as in (2) is normally feedback linearizable if, and only if, there exists a diffeomorphism $T: D \rightarrow \mathbb{R}^{n}$ such that $D_{z}=T(D)$ contains the origin, and the change of variables $z=T(x)$ and a nonsingular matrix $P(x) \in \mathbb{R}^{n \times n}$ that transform the system (2) into (11) according with Definition 4, such that

$$
\begin{aligned}
E_{c} \frac{\partial T(x)}{\partial x} & =P(x) E(x) \\
A_{c} z-B_{c} \Gamma(x) \alpha(x) & =P(x) f(x) \\
B_{c} \Gamma(x) & =P(x) g(x)
\end{aligned}
$$

Proof. The necessity come from Definition 4. By (11) we have:

$$
\begin{aligned}
& E_{c} \dot{z}=A_{c} z-B_{c} \Gamma(x) \alpha(x)+B_{c} \Gamma(x) u \\
& E_{c} \frac{\partial T}{\partial x} \dot{x}=A_{c} T(x)-B_{c} \Gamma(x) \alpha(x)+B_{c} \Gamma(x) u
\end{aligned}
$$

then, if (11) is state-equivalent to (2), follows from Definition 1:

$$
\begin{aligned}
& P^{\prime}(x) E^{\prime}(T(x)) \frac{\partial T(x)}{\partial x}=E(x) \\
& P^{\prime}(x)\left(A_{c} T(x)-B_{c} \Gamma(x) \alpha(x)+B_{c} \Gamma(x) u\right)=f(x)+g(x) u
\end{aligned}
$$

thus, $P^{\prime}(x)$ have inverse and we can write $P^{\prime}(x)^{-1}=P(x)$. Multiplying both sides by $P(x)$, we have (14).

For sufficiency, suppose that $T(x)$ is a smooth solution for (14) and $P(x)$ and $\partial T / \partial x$ are nonsingular. Then one can select $z=T(x)$ as a state-coordinate transformation and (2) can be written as (11). This concludes the proof.

Before using the conditions presented in (14), one must define $E_{c}, A_{c}$ and $B_{c}$ which must be chosen such that the obtained system $\left(E_{c}, A_{c}, B_{c}\right)$ be regular and impulsive-free as the original system (1). Thus $\operatorname{rank}\left(E_{c}\right)=\operatorname{rank}(E(x))=$ $r$ has to be assured. The parameters $r, n$ and $m$ define a controllable linear descriptor system using the structures presented in Lemma 3. The controllability indices and the numbers can be choosed arbitrarily. If $c_{i j}^{k}=0$ and $d_{i j}^{k}=0$, the conditions presented in (14) are more easier to solve, however their choice is also arbitrary.

The application of Proposition 5 generates a differential and algebraic equations system which must be solved to obtain $\Gamma(x), T(x)$ and $\alpha(x)$. This parameters are used to compute the control law (12) applied to linearize and stabilize the system. The singularity of $P(x)$ and $\Gamma(x)$ could be an indicator for the domain of attraction of the closed-loop system. $T(x)$ could be confirmed as a local diffeomorphism with the Inverse Function Theorem presented below:

Theorem 6. (Kass and Vos, 1997). Let $D$ be open in $\mathbb{R}^{n}$ and $T: D \rightarrow \mathbb{R}^{n}$ be a smooth function. If for $x_{0} \in D$, the derivative matrix of $T, d T$, is of full rank $n$, then there exist and open neighborhood $U$ of $x_{0}$ such that $T$ is injective mapping of $U$ onto its image $T(U)$ and its inverse mapping $T^{-1}$ is a smooth function on $f(U)$. In this case, the derivative of $T^{-1}$ at $y_{0}=T\left(x_{0}\right)$ is $d T^{-1}\left(y_{0}\right)=\left(d T\left(x_{0}\right)\right)^{-1}$, that is, it is the inverse of the matrix $d T\left(x_{0}\right)$.

As a consequence of Theorem 6, a function is a local diffeomorphism if its Jacobian is nonsingular.

Thus, we are able to design the control law $v(z)$ in the linear system in (9) via any linear control design technique. For example, one can perform a finite pole allocation in the left half of the complex plane with a state feedback control law $v(z)=-K z$ for the transformed system ensuring $\operatorname{Re}(\lambda)<0$ for the roots of (Yang et al., 2013b):

$$
\operatorname{det}\left(\lambda E_{c}-\left(A_{c}-B_{c} K\right)\right)=0
$$

The stability of the nonlinear closed-loop descriptor system as (13) can be checked using the following theorem.

Theorem 7. (Yang et al., 2013a). Suppose that there exists a function $V(y): \mathbb{R} \rightarrow \mathbb{R}^{+}$with $y=E x$, satisfying the following properties:

(1) $V(0)=0$;

(2) $V$ is continuously differentiable in $\mathbb{R}^{n}$ and twice continuously differentiable in a neighborhood of the origin;

(3) $V(y)>0, \forall y \neq 0$

(4) $\lim _{\|y\| \rightarrow \infty^{+}} V(y) \rightarrow+\infty$;

(5) $(\partial / \partial y) V=\hat{V}^{T}(x) E$ for some function $\hat{V}: \mathbb{R}^{n} \rightarrow \mathbb{R}^{n}$;

(6) $\dot{V}=\hat{V}^{T}(x) f(x)<0, \forall x \neq 0$;

(7) $E^{T} \hat{V}_{x}=\hat{V}_{x}^{T} E \geq 0$, where $\hat{V}_{x}$ denotes the Jacobian of $\hat{V}$;

then the system (2) is globally asymptotically stable of index one ${ }^{2}$.

The proposed controller design procedure and its application can be stated as:

(1) Choose $E_{c}, A_{c}$ and $B_{c}$ according with Lemma (3) and (10). This choice may not to be unique.

(2) Solve the equations given in Proposition 5 to obtain $T(x), \Gamma(x), \alpha(x)$ and $P(x)$ if the number of variables are less than the number of equations to solve. Otherwise, this procedure do not is applicable to the problem.

(3) Check if $T(x)$ is a local diffeomorphism by using Theorem 6 and the singularity of $P(x)$ and $\Gamma(x)$.

(4) Design a linear control law $v(z)$ for the system $\left(E_{c}, A_{c}, B_{c}\right)$ as in $(9)$, according your design requirements.

(5) Check the stability of the closed-loop nonlinear system as in (13) with Theorem 7.

(6) Apply the control law (12).

\footnotetext{
2 According to Yang et al. (2013a), a system as in (2) is of index one if the constant coefficient system

$$
E \dot{w}=f_{x}(\hat{x}) w
$$

is regular and impulse-free for all $\hat{x}$ in a neighborhood of the equilibrium point $x=0$ where $f_{x}$ is the Jacobian matrix.
} 


\section{NUMERICAL EXAMPLES}

\subsection{Example 1}

Consider a nonlinear descriptor system as described in (1):

$$
\left[\begin{array}{cc}
1 & 0 \\
1 & x_{2}^{2}+1
\end{array}\right] \dot{x}=\left[\begin{array}{cc}
0 & 1 \\
2 & x_{2}
\end{array}\right] x+\left[\begin{array}{l}
0 \\
1
\end{array}\right] u
$$

Notice that the matrix $E(x)$ is regular and positive definite for all $x$.

First, in order to apply the proposed methodology, we define a controllable linear descriptor system as in (9) by choosing $E_{c}, A_{c}$ and $B_{c}$ according to Lemma 3. In this case, we have for $n=2, m=1$ and $r=2$ :

$$
E_{c}=\left[\begin{array}{ll}
1 & 0 \\
0 & 1
\end{array}\right], \quad A_{c}=\left[\begin{array}{ll}
0 & 0 \\
1 & 0
\end{array}\right] \quad \text { and } \quad B_{c}=\left[\begin{array}{l}
1 \\
0
\end{array}\right] .
$$

where $c_{11}^{1}=c_{11}^{K_{1}=2}=0$.

Applying the Proposition 5, we obtain the following parameters with $P(x)=\left[\begin{array}{cc}-\left(x_{2}^{2}+1\right)^{-1} & \left(x_{2}^{2}+1\right)^{-1} \\ 1 & 0\end{array}\right]$ :

$$
\begin{aligned}
\Gamma(x) & =\frac{1}{x_{2}^{2}+1} \\
\alpha(x) & =x_{2}-2 x_{1}-x_{2}^{2} \\
T(x) & =\left[\begin{array}{l}
T_{1}(x)=x_{2} \\
T_{2}(x)=x_{1}
\end{array}\right]
\end{aligned}
$$

which transform the system (17) according with (11). By Theorem $6, T(x)$ is a local diffeomorphism.

Allocating the finite poles of the resulting linear descriptor system as described in (9) at $-2 \pm 3 j$ by solving (16), we have the following state feedback control law:

$$
v(z)=-\left[\begin{array}{ll}
4 & 13
\end{array}\right] z
$$

At this point, we have all the elements that define the nonlinear control law (12), which is defined, in this case, by the parameters presented in (19) and (20). It is concluded that the system (17) is normally feedback linearizable as in (11) with data given in (18) and (19).

The Theorem 7 can be used to check the stability of closedloop system as in (13). The following function candidate satisfies all the necessary conditions to conclude that global asymptotically stability in the sense of Lyapunov holds.

$$
V_{1}(E x)=x^{T} E(x)^{T} W E(x) x=x^{T}\left[\begin{array}{rr}
17 & 1 \\
1 & 1
\end{array}\right] x
$$

The results of a simulation of closed-loop of (17) with control law as in (12) and an initial condition $x_{0}=\left[\begin{array}{ll}3 & -2\end{array}\right]^{T}$ are depicted in Figure 1. As expected by the choice of the finite poles, we have under damped trajectories for the state $x$. The evolution of the proposed Lyapunov function $V_{1}(E x)$ is presented in Figure 2.

\subsection{Example 2}

Consider the following nonlinear descriptor system adapted from Lin et al. (2006) and described as in (1):

$$
\left[\begin{array}{lll}
1 & 0 & 0 \\
0 & 1 & 0 \\
0 & 0 & 0
\end{array}\right] \dot{x}=\left[\begin{array}{ccc}
0 & 1 & 0 \\
0 & 0 & \left(a x_{2}^{2}+1\right) \\
c & -b x_{2}^{2} & \left(-1-a \cos \left(x_{1}\right)\right)
\end{array}\right] x+\left[\begin{array}{ll}
0 & 0 \\
0 & d \\
d & 0
\end{array}\right] u
$$

with $a=0.1, b=-0.1$ and $c=d=1$.

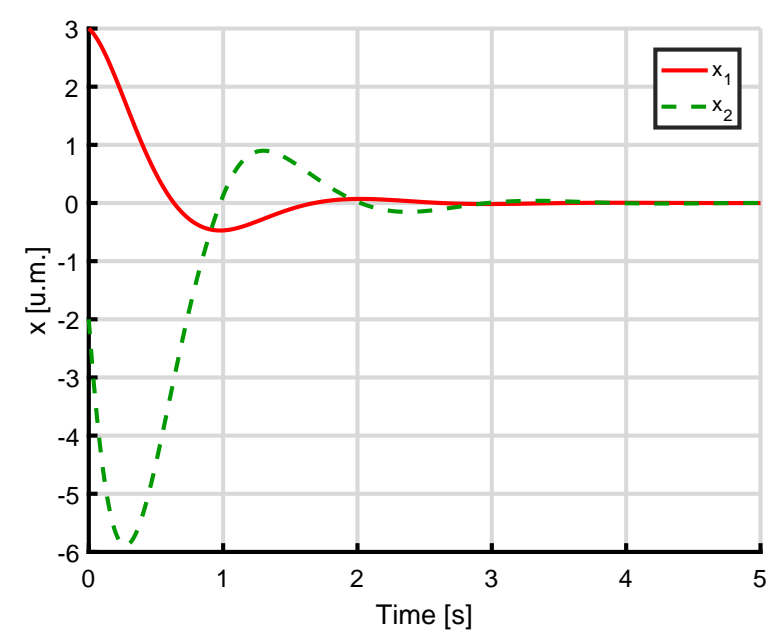

(a) $x(t)$

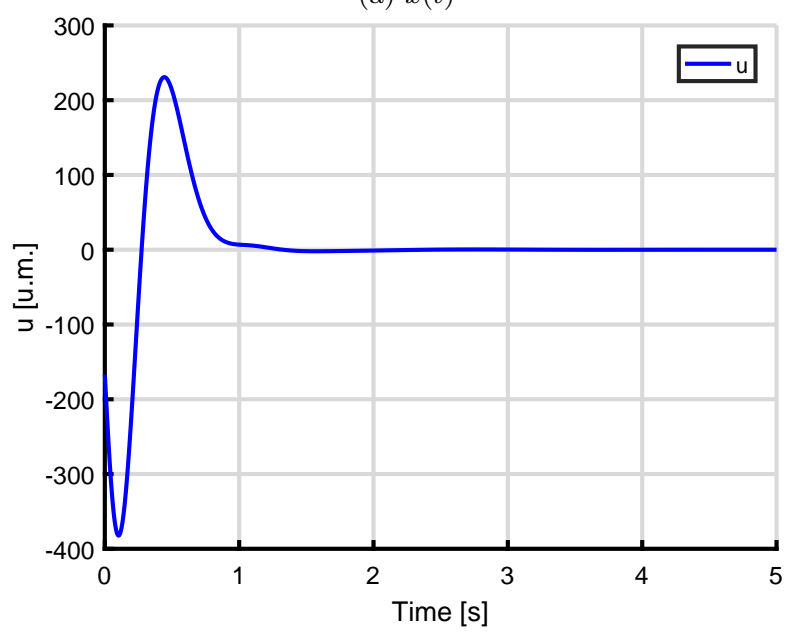

(b) $u(t)$

Figure 1. Trajectories for the closed-loop system (17) with the control law $u$ as in (12) with parameters in (19) and (20) for initial condition $x_{0}=\left[\begin{array}{ll}3 & -2\end{array}\right]^{T}$.

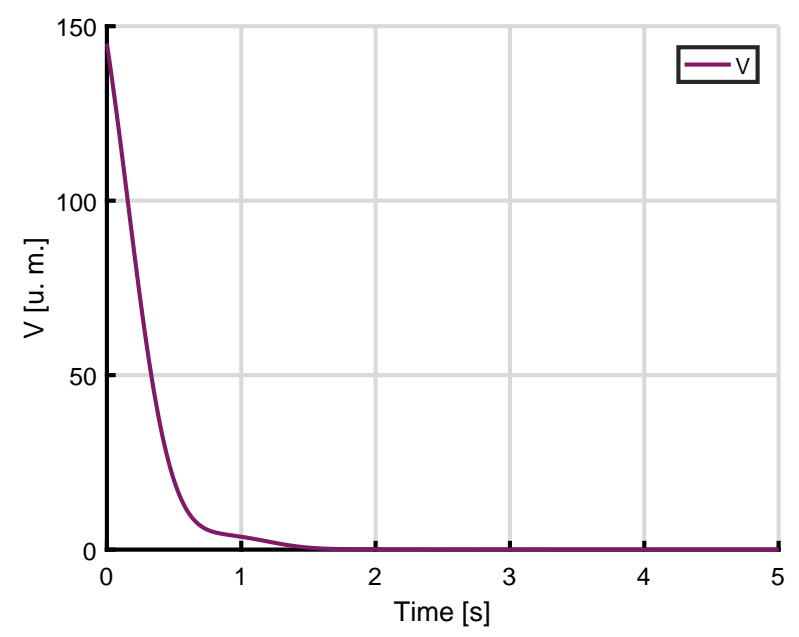

Figure 2. Evolution in time of (21) for initial condition $x_{0}=\left[\begin{array}{ll}3 & -2\end{array}\right]^{T}$ of system (17) in closed-loop.

By Lemma 3 and according (10), we have for $n=3, m=2$ and $r=2$ : 


$$
E_{c}=\left[\begin{array}{lll}
1 & 0 & 0 \\
0 & 1 & 0 \\
0 & 0 & 0
\end{array}\right], \quad A_{c}=\left[\begin{array}{ccc}
0 & -1 & 0 \\
1 & 0 & 0 \\
0 & 0 & 1
\end{array}\right] \quad \text { and } \quad B_{c}=\left[\begin{array}{ll}
1 & 0 \\
0 & 0 \\
0 & 1
\end{array}\right] .
$$

where $c_{11}^{1}=-1$ and $c_{11}^{K_{1}=2}=0$.

Then, applying Proposition 5 we obtain:

$$
\begin{aligned}
\Gamma(x) & =\left[\begin{array}{ll}
1 & 1 \\
1 & 0
\end{array}\right] \\
\alpha(x) & =\left[\begin{array}{c}
-x_{1}-0.1 x_{2}^{3}+0.1 x_{3} \cos \left(x_{1}\right) \\
-0.1 x_{2}^{2} x_{3}-x_{1}
\end{array}\right] \\
T(x) & =\left[\begin{array}{c}
T_{1}(x)=x_{2} \\
T_{2}(x)=x_{1} \\
T_{3}(x)=-x_{3}
\end{array}\right]
\end{aligned}
$$

with

$$
P(x)=\left[\begin{array}{lll}
0 & 1 & 1 \\
1 & 0 & 0 \\
0 & 0 & 1
\end{array}\right]
$$

By its Jacobian matrix we prove that $T(x)$ is a local diffeomorphism, according with Theorem 6 .

Thus, if we choose the closed-loop poles as $\lambda_{1}=-1$ and $\lambda_{2}=-2$ for the linear system, by (16) we have:

$$
v(z)=-K z=-\left[\begin{array}{lll}
4 & 2 & 1 \\
1 & 1 & 2
\end{array}\right] z
$$

Then the system $\Sigma_{2}$ is normally feedback linearizable by the nonlinear control law as in (12) with (23) and (24). In the same manner as Example 1, one can use the Theorem 7 to check the stability of the closed-loop system which have the form of (13). The function candidate (25) satisfies all the necessary conditions to conclude that global asymptotically stability in the sense of Lyapunov holds.

$$
V_{2}(y)=y^{T}\left[\begin{array}{ccc}
2 & 0.5 & 0 \\
0.5 & 1 & 0 \\
0 & 0 & 0
\end{array}\right] y
$$

Figure 3 depicts the trajectories of the system (22) with the designed closed-loop with the initial condition $x_{0}=$ $\left[\begin{array}{lll}1.2 & -0.8 & 0.4\end{array}\right]^{T}$. Figure 4 depicts the evolution of $(25)$ according with the trajectories presented in Figure 3.

\section{FURTHER DISCUSSIONS}

The proposed method depends on the solution of a differential and algebraic equation system defined by (14). The number of variables to find depends on the order of the state vector $n$ and the number of inputs $m$ in (1).

The process to solve the equations in (14) requires to guess some parameters, which implies that we could have infinite solutions that linearize the system. However, the integration steps to solve the differential parts bring difficulties and may prevent to get a batch solution.

If a solution is found, the controller design takes advantage of all the design facilities of a linear controller. It is possible to define the settling time and the percent overshoot, and if the solution is global, there is no sensitivity of these parameters to the initial conditions.

With $m=1, n>2$ and $\operatorname{rank}(E(x))=n$, the number of variables are less than the number of equations to solve. Then, in this case, the proposed transformation of

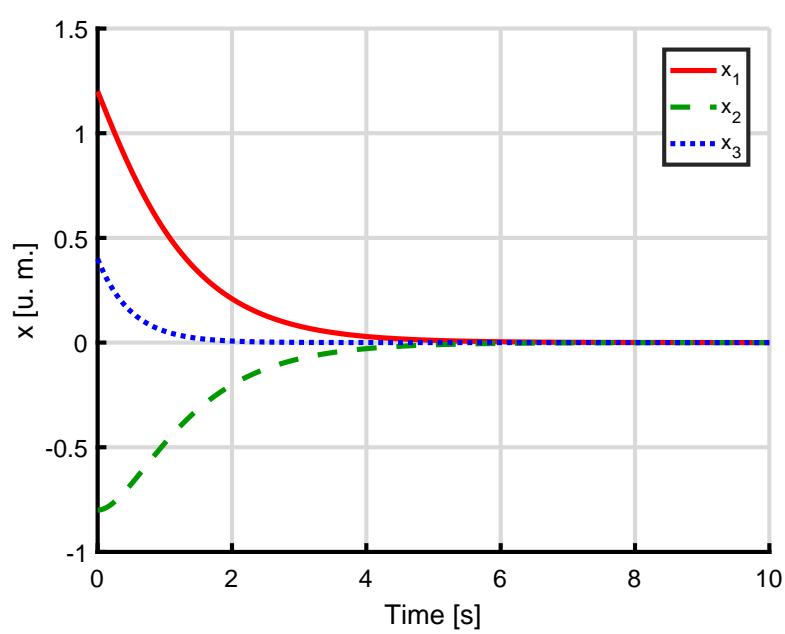

(a) $x(t)$

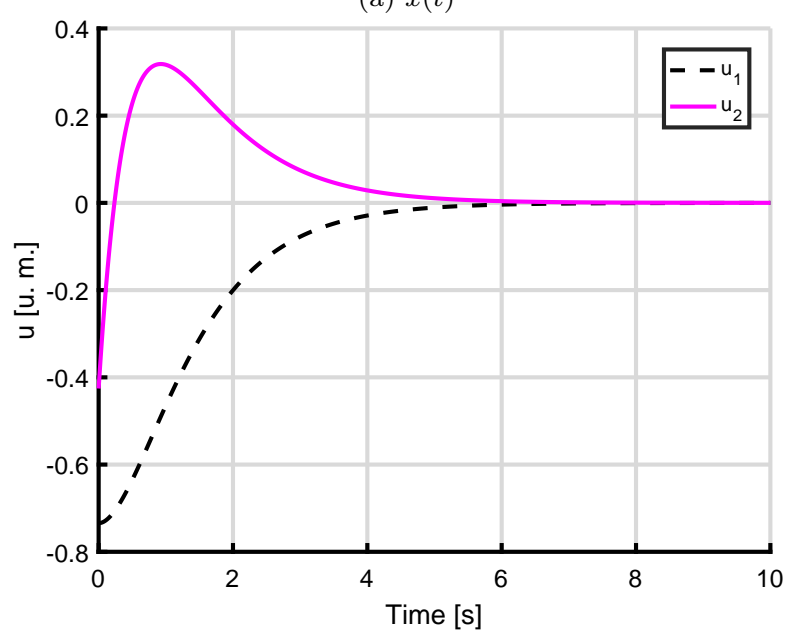

(b) $u(t)$

Figure 3. Trajectories for the closed-loop system (22) with the control law $u$ as in (12) with parameters in (23) and (24) for initial condition $x_{0}=\left[\begin{array}{lll}1.2 & -0.8 & 0.4\end{array}\right]^{T}$.

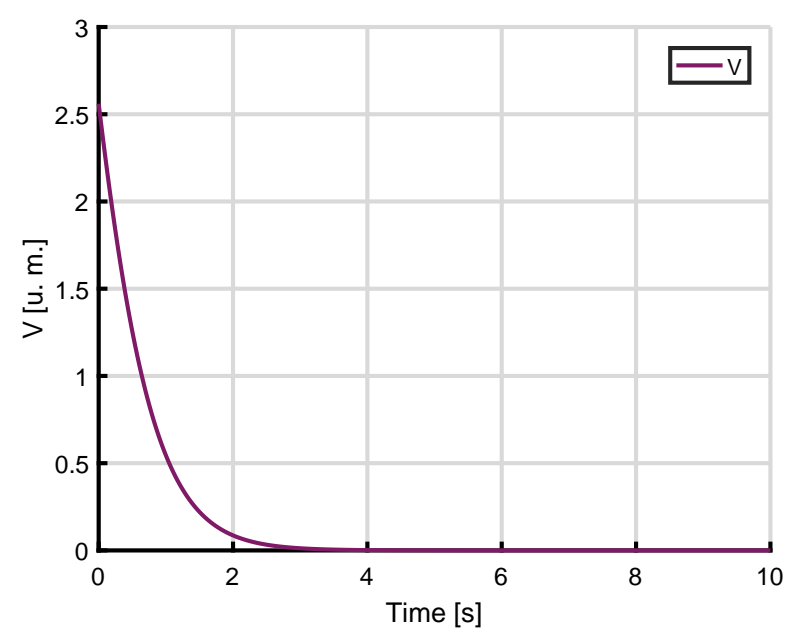

Figure 4. Evolution in time of (25) for initial condition $x_{0}=\left[\begin{array}{lll}1.2-0.8 & 0.4\end{array}\right]^{T}$ of system $(22)$ in closed-loop.

Proposition 5 does not exist, because the process implies to obtain some variables in not a unique way. 


\section{CONCLUSIONS}

This paper has proposed one method of feedback linearization for nonlinear descriptor systems. We present conditions for transform the system and design a control law that linearizes and stabilizes the system. Numerical examples have been presented to illustrate the effectiveness of the proposed method. Future works include to study the solvability of differential and algebraic equations as in (14) generated by the conditions proposed.

\section{ACKNOWLEDGMENTS}

This work has been supported by the Brazilian agencies CAPES, CNPq and FAPEMIG.

\section{REFERENCES}

Blandeau, M., Estrada-Manzo, V., Guerra, T., Pudlo, P., and Gabrielli, F. (2018). Fuzzy unknown input observer for understanding sitting control of persons living with spinal cord injury. Engineering Applications of Artificial Intelligence, 67, 381-389. doi:10.1016/j.engappai.2017. 09.016.

Boukas, T. and Habetler, T. (2003). Exact feedback linearization with state derivative feedback for high-performance field-oriented induction motor speed/position control. In 38th IAS Annual Meeting on Conference Record of the Industry Applications Conference, 2003, volume 3, 1990-1996. IEEE. doi:10.1109/ IAS.2003.1257840.

Dai, L. (1989). Singular Control Systems. Springer Berlin Heidelberg, 1 edition.

Guelton, K., Delprat, S., and Guerra, T.M. (2008). An alternative to inverse dynamics joint torques estimation in human stance based on a Takagi-Sugeno unknowninputs observer in the descriptor form. Control Engineering Practice, 16(12), 1414-1426. doi:10.1016/j. conengprac.2008.04.002.

Helmke, U. and Shayman, M. (1989). A canonical form for controllable singular systems. Systems \& Control Letters, 12(2), 111-122. doi:10.1016/0167-6911(89)90003-0.

Kass, R.E. and Vos, P.W. (1997). Geometrical Foundations of Asymptotic Inference. John Wiley \& Sons, Inc. doi: 10.1002/9781118165980.

Kawaji, S. and Taha, E. (1994). Feedback linearization of a class of nonlinear descriptor systems. In Proceedings of the 33rd IEEE Conference on Decision and Control, volume 4, 4035-4037. IEEE. doi:10.1109/CDC.1994. 411576.

Lin, C., Wang, Q.G., and Lee, T.H. (2006). Stability and stabilization of a class of fuzzy time-delay descriptor systems. IEEE Transactions on Fuzzy Systems, 14(4), 542-551. doi:10.1109/tfuzz.2006.876738.

Luenberger, D.G. (1978). Time-invariant descriptor systems. Automatica, 14(5), 473-480. doi:10.1016/ 0005-1098(78)90006-7.

Newcomb, R.W. and Dziurla, B. (1989). Some circuits and systems applications of semistate theory. Circuits, Systems, and Signal Processing, 8(3), 235-260. doi:10. $1007 /$ bf01598413.

Schulte, H. and Guelton, K. (2009). Descriptor modelling towards control of a two link pneumatic robot manipulator: A T-S multimodel approach. Nonlinear Analysis:
Hybrid Systems, 3(2), 124-132. doi:10.1016/j.nahs.2008. 11.003.

Slotine, J.J.E. and Li, W. (1991). Applied Nonlinear Control. Prentice Hall, Englewood Cliffs, N.J.

Spong, M. (1994). Partial feedback linearization of underactuated mechanical systems. In Proceedings of IEEE/RSJ International Conference on Intelligent Robots and Systems (IROS'94), volume 1, 314-321. IEEE. doi:10.1109/IROS.1994.407375.

Xiaoping, L. (1993). On Linearization of Nonlinear Singular Control Systems. 1993 American Control Conference, 26(2), 2284-2287. doi:10.23919/ACC.1993. 4793293.

Yang, C., Sun, J., Zhang, Q., and Ma, X. (2013a). Lyapunov stability and strong passivity analysis for nonlinear descriptor systems. IEEE Transactions on Circuits and Systems I: Regular Papers, 60(4), 1003-1012. doi: 10.1109/tcsi.2012.2215396.

Yang, C., Zhang, Q., and Zhou, L. (2013b). Stability Analysis and Design for Nonlinear Singular Systems. Springer Berlin Heidelberg, 1 edition. doi:10.1007/ 978-3-642-32144-3. 